\title{
Electric passenger car transport and passenger car life cycle inventories in ecoinvent version 3
}

\author{
Andrea Del Duce • Marcel Gauch • Hans-Jörg Althaus
}

Received: 22 April 2013 / Accepted: 13 August 2014 / Published online: 19 September 2014

(C) Springer-Verlag Berlin Heidelberg 2014

\begin{abstract}
Purpose Due to the large environmental challenges posed by the transport sector, reliable and state-of-the art data for its life cycle assessment is essential for enabling a successful transition towards more sustainable systems. In this paper, the new electric passenger car transport and vehicle datasets, which have been developed for ecoinvent version 3 , are presented.

Methods The new datasets have been developed with a strong modular approach, defining a hierarchy of datasets corresponding to various technical components in the vehicle. A vehicle is therefore modelled by linking together the various component datasets. Also, parameters and mathematical formulas have been introduced in order to define the amount of exchanges in the datasets through common transport and vehicle characteristics. This supports users in the choice of the amount of exchanges and enhances the transparency of the dataset.

Results The new transport dataset describes the transport over $1 \mathrm{~km}$ with a battery electric passenger car taking into account the vehicle production and end of life, the energy consumption due to the use phase, non-exhaust emissions, maintenance and
\end{abstract}

Responsible editor: Rainer Zah

Electronic supplementary material The online version of this article (doi:10.1007/s11367-014-0792-4) contains supplementary material, which is available to authorized users.

\section{A. Del Duce $(\bowtie)$}

Quantis, Reitergasse 11, 8004 Zurich, Switzerland

e-mail: andrea.delduce@quantis-intl.com

A. Del Duce $\cdot$ M. Gauch $\cdot$ H.-J. Althaus

Empa, Life Cycle Assessment and Modelling Unit, Überlandstrasse

129, 8600 Dübendorf, Switzerland

H.-J. Althaus

Swisscleantech, Reitergasse 11, 8004 Zurich, Switzerland road infrastructure. The dataset has been developed and is suitable for a compact class vehicle.

Conclusions A new electric passenger car transport dataset has been developed for version 3 of the ecoinvent database which exploits modularisation and parameters with the aim of facilitating users in adapting the data to their specific needs. Apart from the direct use of the transport dataset for background data, the various datasets for the different components can also be used as building blocks for virtual vehicles.

Keywords Ecoinvent · Electric mobility · Electric vehicles · Life cycle assessment $\cdot$ Sustainable transportation systems

\section{Introduction}

The transport sector is responsible for a number of crucial environmental challenges. In Europe, for example, it causes a large share of air pollution, while in 2006, it consumed about one third of all final energy (most of which of fossil origin) and produced around $20 \%$ of the total European greenhouse gas (GHG) emissions (Mahieu 2009). Moreover, with more than $70 \%$ modal share in passenger transport (Huggins 2009), passenger cars are a major contributor in the environmental impacts caused by transport systems. Since electric passenger cars can be run with electricity obtained from renewable energy sources, there is the perception that they can play a key role in the transition towards more sustainable transport systems (see, for example, Grünig et al. 2011). However, in order to avoid that unexpected shifting of burden may offset the potential benefits of electric vehicles if these were to reach high levels of penetration, a holistic approach based on reliable and up-to-date data is required.

Ecoinvent version 2 proposes a variety of passenger car transport datasets for life cycle assessment (LCA) covering various technologies and fuel types. These range from internal 
combustion engine (ICE) vehicles in their petrol and diesel versions and considering different Euro standards to natural gas vehicles as well as battery electric vehicles (BEVs) and hybrid passenger cars. While most of the data is described and documented in Spielmann and Bauer et al. (2007), the core data on the passenger car infrastructure is mostly based on Schweimer and Levin (2000).

In using these datasets, practitioners are confronted with following challenges. First, the transport datasets are based on a very specific vehicle. If a practitioner is interested, for example, in a vehicle with a different weight, then postprocessing of the data will be required or he will be forced to adapt his study to the same vehicle as in the database. Also, the datasets describing the production of the passenger cars are built to a large extent as a black box in which, with a few exceptions, all the materials, resources and emissions required for building the vehicle as a whole are listed without specifying what entries relate to which components within the vehicles. This hinders the understanding of where the hot spots in the production of a vehicle lie. Similarly, the "operation" files describing the resources and emissions involved in the use of the vehicles take into account all the exhaust emissions (when appropriate) for a specific consumption of the vehicle and the non-exhaust emissions without clearly differentiating their origin (i.e. tyre, brake or road). Again, if a practitioner is interested in a transport service of a vehicle with a different consumption or with a different share of non-exhaust emissions, post-processing of the data by the user will be needed. However, not all users may be familiar with how to modify the default data in order to correctly model their desired situation of interest. Hence, in order to obtain a more flexible and transparent use of transport datasets, a different structure is required.

The Technology-Centred Electric Mobility Assessment (THELMA) project aimed at assessing the implications of a widespread use of electric vehicles for private passenger transport in Switzerland (THELMA 2010). Within this project, LCA was used to compare the environmental impacts of a broad spectrum of current technology vehicles which required to develop a large number of new transport datasets. In developing these datasets, a high degree of modularity was used in order to facilitate the modelling of new virtual vehicles by linking datasets of the components which are within a passenger car. Also, parameterisation was introduced, for example through the mass of the vehicle, so that transport datasets could cover a class of vehicles with variable weight instead of a single, specific model. The datasets developed within the THELMA project were then used as the base for the new passenger car transport datasets in ecoinvent version 3 . This paper describes the transport dataset for the battery electric passenger car as well as the datasets covering the production and end of life of the passenger cars which have been accepted for ecoinvent $\mathrm{v} 3$.

\section{Methodology}

2.1 The ecoinvent v2 electric passenger car transport dataset

Figure 1 shows the structure of the ecoinvent v2.2 transport datasets (DS) for the battery electric passenger car.

The structure relies on five main puts: the dataset describing the resources and emissions involved in the production of the electric passenger car, in its operation, maintenance and disposal and the datasets which take into account road infrastructure. As mentioned in the introduction, this structure brings following challenges. First of all, the dataset addressing the production of the electric passenger car was developed for one specific vehicle, the compact class Volkswagen Golf described in Schweimer and Levin (2000). Further, this dataset describes the production of the electric vehicle bringing together all the resources and emissions which are necessary for building it as a whole as schematised in Fig. 2.

The only exceptions are two inputs separately describing the production of the electric motor and of the lithium-ion battery built within the vehicle. The main disadvantages of this structure are two. First of all, this dataset describes a very specific vehicle. Unless the user is interested in exactly the same electric passenger car, he will have to change the various entries in the vehicle production dataset in order to adapt the data to his needs. However, not all users might be familiar with how to correctly change the input and outputs in case they want to model a different vehicle. Then, since to a good extent the dataset describes the production of the vehicle as if it was a single device, it is not possible to analyse how the various parts within the vehicles contribute to the overall impacts.

A further challenge in using the transport dataset schematised in Fig. 1 lies in the operation file. This dataset was derived for the vehicle corresponding to the one of the production dataset which means that it describes the energy consumption and emissions of that specific vehicle. Hence, again, if a user is interested in an operation dataset for a slightly different vehicle or the same vehicle but testing a different energy consumption, he will have to analyse how to change each single exchange in the operation dataset. Doing this is also complicated by the fact that the emissions are listed all together without further subdividing these according to their nature-i.e. non-exhaust emissions from tyre, brake or road wear and exhaust emissions in case of ICE vehicles, making it difficult for users to understand how to adapt the data to their needs.

Hence with respect to, both, the vehicle production dataset and the operation dataset, a key issue in the limitations of the transport datasets for the electric passenger car in ecoinvent v2.2 is the one of flexibility, since adapting the datasets to situations different to the ones originally depicted is not straightforward and may even lead to errors in case the user 


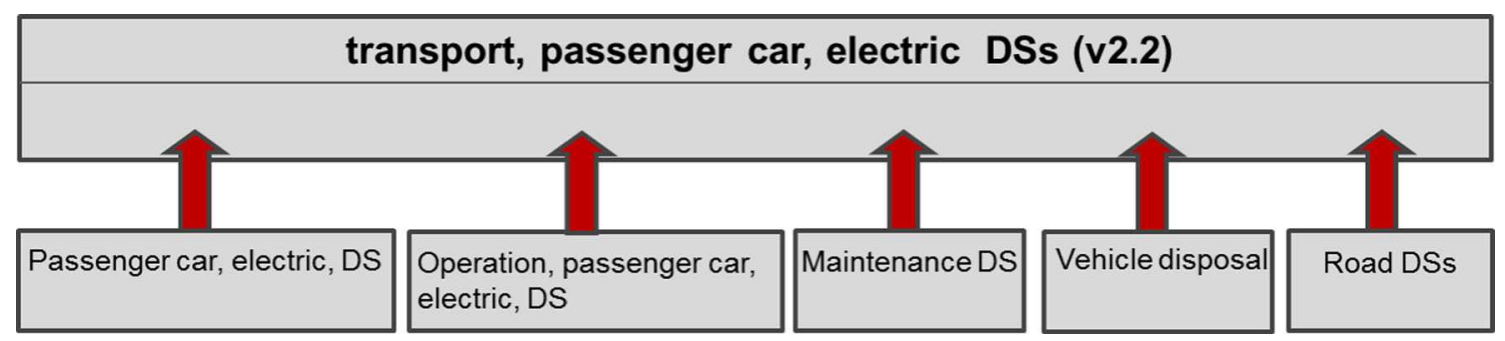

Fig. 1 Structure of the ecoinvent v2.2 passenger car transport datasets

has no in-depth knowledge of the framework behind the given datasets. Overall, in developing the new transport dataset for the electric passenger car, the main idea was not to deliver a dataset describing one specific vehicle, but to structure it so that it could be more easily adapted for a range of vehicles belonging to the class of compact size passenger cars.

In addressing the differences between the ecoinvent v 2.2 electric passenger car transport dataset and the one for version 3 , it is also important to mention a key change in the new version of the ecoinvent database itself and what influence it brought on the datasets therein. Based on the methodological guidelines for version 2 of the database (Frischknecht et al. 2007), the passenger car transport datasets, both for ICE vehicles or the electric version, do not take into account the quantities of recycled materials which can be extracted from the vehicle during the end-of-life phase. The dataset containing information about the disposal of the vehicle mainly includes data on the waste residues from the disposal process which are sent to incineration, while the effectively recycled materials like steel or copper are cut off and their quantities are not reported (Spielmann et al. 2007). In the new version of the database, the end of life of products is treated differently. As described in detail in Weidema et al. (2013), the new database stores the data as multi-output processes (of which disposal processes are a key example) so that multi-functionality can be solved either through an allocation procedure or through

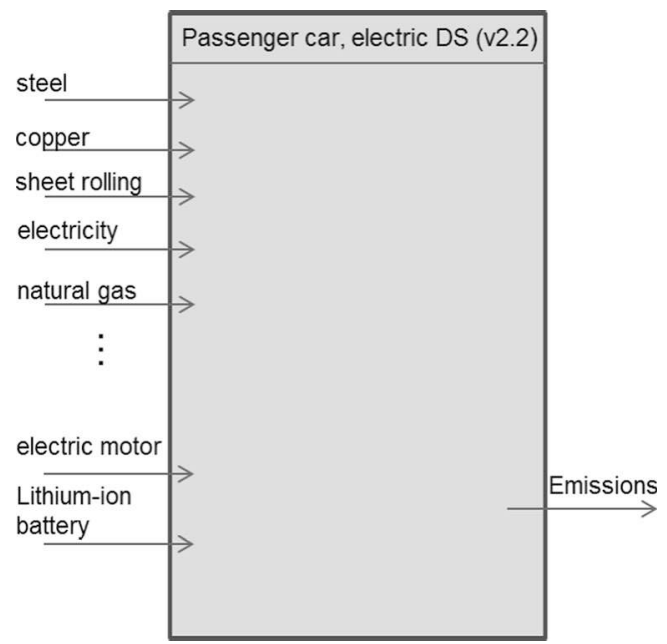

Fig. 2 Main inputs and outputs of the dataset describing the production of the electric passenger car in version 2.2 of the ecoinvent database system expansion. Recycling processes are therefore typically modelled through treatment activities. For the case of the end of life of vehicles, this requires to identify the resources and impacts related to the treatment of a used vehicle and to quantify the materials which are won back during the disposal stage and that can be recycled in the production of other products. Hence, the development of the new electric passenger car transport datasets also required, as will be described in more detail below, to have a closer look at the end of life of passenger cars.

\subsection{Modularisation in the new datasets}

In order to provide ecoinvent v3 users with a more flexible electric passenger transport dataset, it was first necessary to update and change the structure behind the new passenger car production dataset. The main aim was to produce a passenger car dataset which could be easily adapted for the transport dataset so as to cover a range of vehicles within the compact class size. A key feature for achieving this result was to enhance the modularity of the dataset structure with respect to the various components or assemblies in the car. In other words, instead of having a single dataset describing all the materials, energy resources and emissions required for the production of the vehicle, the new dataset is based on submodules describing the production of the key technical components in an electric vehicle. As will be shown in the following paragraphs, this structure allows to obtain an electric passenger car transport dataset in which the mass of the vehicle which is analysed can be easily changed. Moreover, users more interested in life cycle inventory data of vehicles or of vehicle components, rather than the corresponding transport dataset, have now a library of modules with which they can model the vehicle which best suits their needs or just focus on a specific component within the vehicle. Since within the THELMA project, electric passenger cars were compared to various ICE vehicles, the same restructuring of the datasets was made also for the production of diesel, petrol and natural gas vehicles.

In developing a modular dataset, it was first necessary to define a convenient decomposition of the vehicle into modules corresponding to key technical functionalities. The first subdivision of the vehicle was in "glider" and "powertrain". 
The powertrain comprises all the components which are required for generating and transmitting the propulsive energy for the vehicle. For the BEV, these are the electric motor, the battery and the power electronics, which process the electric energy in the system and comprise a converter, an inverter, a power distribution unit (PDU) and a charger. For the ICE vehicle, the powertrain comprises, among other devices, the engine, gearbox and tank. The glider brings together all remaining components of the vehicle which are not strictly related to the propulsion technology (i.e. electric as opposed to ICE): the chassis/body, tyres and wheels, seats, belts, windshield and windows, suspension system, etc. The glider is therefore assumed to be the same for the ICE vehicles and for BEVs. Figure 3 shows the decomposition on which the definition of the new datasets has been based. Because the focus of the THELMA project was on electric mobility, a higher degree of modularity was used for the BEV.

Starting from the decomposition shown in Fig. 3, datasets for each of the modules were derived. Compared to the passenger cars available in ecoinvent version 2, another key difference has been implemented. For the electric passenger car, the lithium-ion battery has been taken out from the dataset describing the production of the vehicle, and it has been located directly into the transport dataset. This was done because the lithium-ion battery still represents one of the most critical components within an electric vehicle, due to its large change margins on capacity improvements and cost reduction (Duleep et al. 2011). Moving the battery from the electric passenger car production dataset into the electric passenger car transport dataset allows users to change more easily its quantity or type, in order to analyse, for example, the impacts deriving from different battery capacities or technologies in the vehicle in the context of designs for various range targets or from different battery replacement strategies.

With respect to the end of life of the vehicle and to the accounting of the recycled materials, following strategy was used in the new modular datasets. The data for the new datasets has been developed starting from the main recycling processes involved in the end of life of a vehicle in Europe and the USA. In this context, it must be noted that, based on Schneider et al. (2010), this scenario may not hold for other countries or for those vehicles which are legally or illegally resold to countries with different and less stringent regulations on the end of life of passenger cars and for which little information is currently available. Following Jody et al. (2010), the first step in the end of life takes usually place in a manual dismantling facility in which re-sellable parts are removed as well as hazardous substances which are then disposed of accordingly. Components which are worth being sent to dedicated recycling facilities are also removed. Based on Hischier (2007), it was assumed that the powertrain of a current technology electric vehicle comprises high-quality electronics and materials and would therefore be sent to a dedicated recycling facility in which it would be manually dismantled in order to extract the metallic cases (steel or aluminium) and send the remaining electronics and printed wiring boards (PWB) to an electronics recycling facility. The rest of the vehicle, which means essentially the glider for the BEV and glider plus ICE powertrain for the ICE vehicle, is sent to a shredding facility. In Fig. 4, the main steps considered for modelling the end of life of the electric passenger car and of the ICE vehicle are summarised.

Following Fig. 4, datasets modelling the infrastructure and/ or recovered materials for, respectively, the manual dismantling of a vehicle, the treatment of the glider, the treatment of the ICE powertrain and the treatment of the BEV's powertrain were developed. The reference flows of these datasets appear as exchanges in the datasets describing the production of the corresponding module. The treatment of the used glider, for example, is an exchange in the dataset describing the production of the glider. In this way, users who are interested in using the glider datasets do not need to calculate by themselves the materials which may be won back, depending on the amount of glider their analysis requires. By choosing the desired
Fig. 3 Schematic decomposition of the vehicle into the modules used for the datasets for a electric and $\mathbf{b}$ vehicles based on ICEs
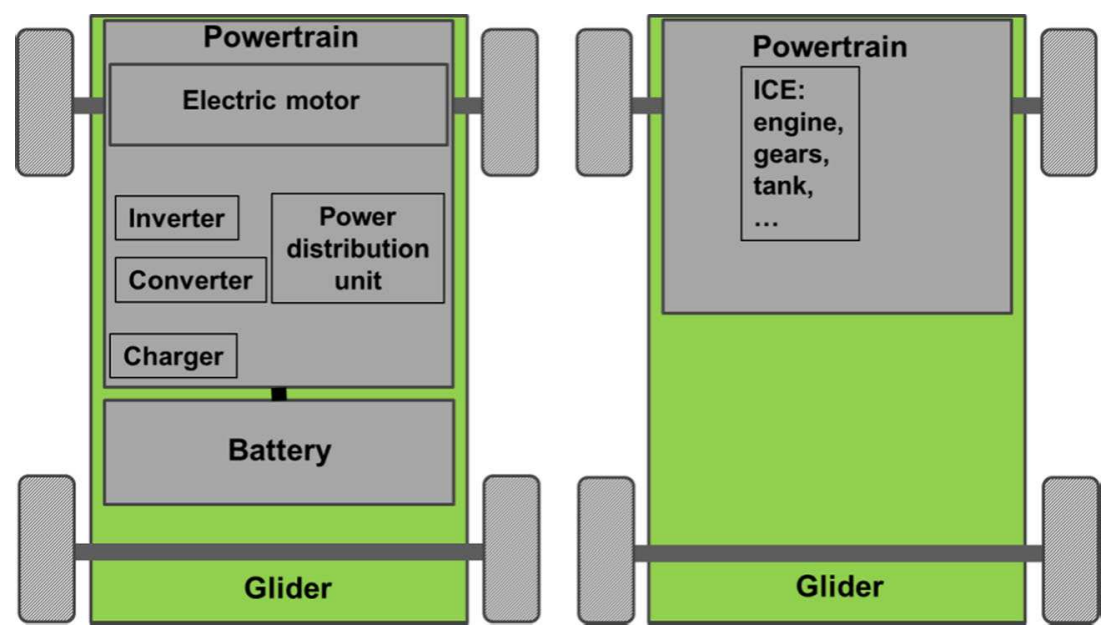
Fig. 4 Main steps of vehicle end of life

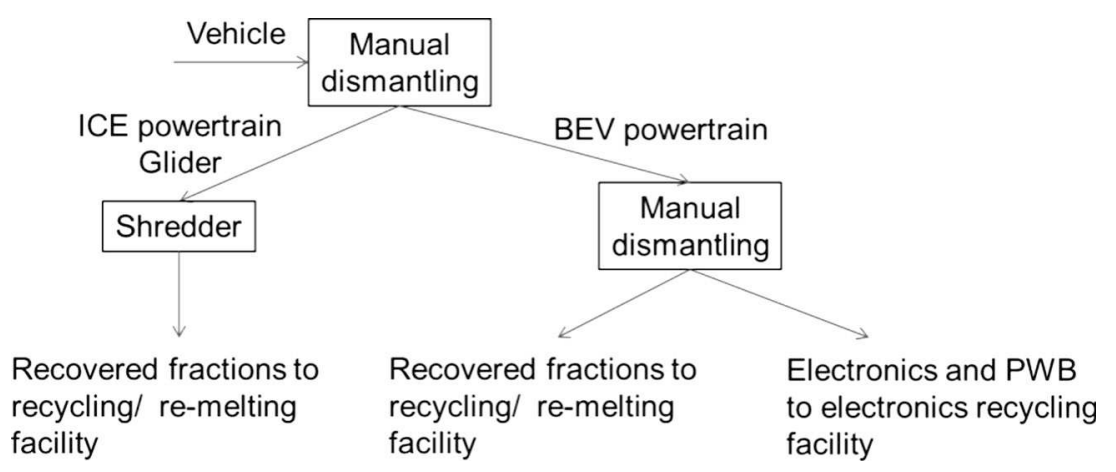

amount of glider, the correct amount of treatment of the used glider will be accounted for automatically. More details on the end-of-life datasets are given in the sections describing the various components. Overall, the complete structure of the electric and ICE passenger car production dataset is summarised in Fig. 5. For the electric vehicle, the dotted "ICE production, passenger car" branch should be ignored, whereas for the two ICE vehicles in the database (diesel and petrol/natural gas), it is the "powertrain production, for electric passenger car" one which should not be considered in the figure.

In Fig. 5, all the framed names of datasets (e.g. "charger production, electric passenger car" or "treatment of used charger") are new datasets which have been specifically prepared for the new electric passenger car transport dataset. The other entries (e.g. "electronic components" or "materials") generally describe datasets which have been taken from the ecoinvent database. Below, details on the derivation of the datasets for the main modules shown in Fig. 5 are given. The complete list of datasets which have been created can be found in the Electronic Supplementary Material.

\subsubsection{Glider production}

The data for the dataset describing the production of the glider is based on literature and was derived from Schweimer and Levin (2000), Althaus and Gauch (2010) and Habermacher (2011). In Schweimer and Levin (2000), the life cycle inventory for the production of a Volkswagen Golf is presented, highlighting the required materials, production energies and the generated wastes. In Althaus and Gauch (2010), the data presented by Schweimer and Levin, who described a Golf A4, was analysed and grouped into two separate modules for the glider and ICE powertrain. Further, looking at the weight and technical specifications of a more modern Golf VI (Volkswagen 2008), Althaus and Gauch scaled the datasets in order to portray a contemporary technology. Overall, the total weight of the Golf A4 is of $1,058 \mathrm{~kg}$, which Althaus and Gauch analysis subdivided into a glider of 783 and $275 \mathrm{~kg}$ for the petrol ICE powertrain (Althaus and Gauch 2010). Similarly, the Golf VI is characterised in its petrol and diesel version by a total weight of, respectively, 1,234 and 1,314 kg, which Althaus and Gauch subdivided in a glider of $913 \mathrm{~kg}$ for

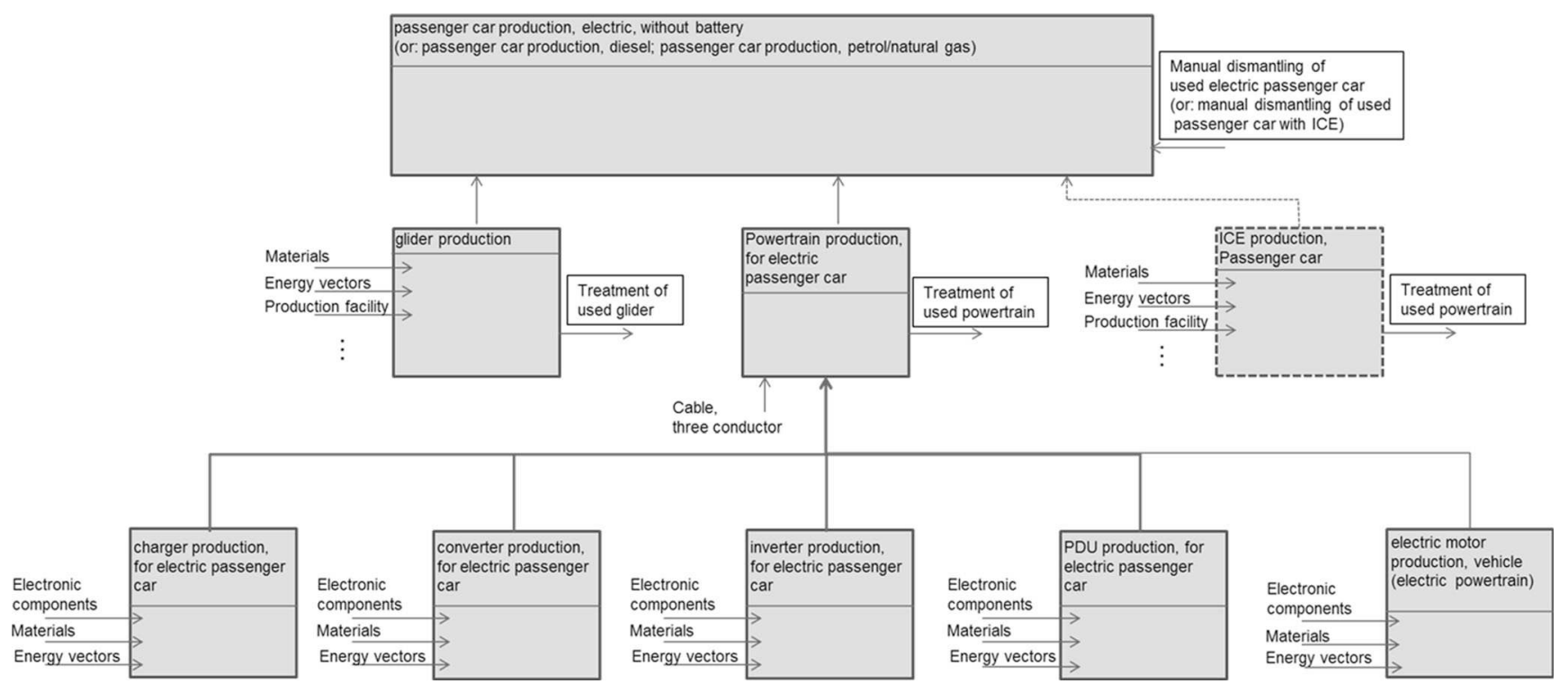

Fig. 5 Structure of the electric and ICE passenger car production dataset. For the electric vehicle, the dotted branch should be neglected, whereas for ICE vehicles, the sub-graph addressing the production of the electric powertrain should not be considered 
both technologies and an ICE powertrain weighting $321 \mathrm{~kg}$ for petrol and $401 \mathrm{~kg}$ for the diesel version. Hence, to model the higher weight of the Golf VI, Althaus and Gauch depicted its glider by taking the dataset derived for the Golf A4 and scaling it linearly by a factor of 1.16 , corresponding to the ratio of the total weight of the Golf VI and A4 in the petrol version. Building on the work of Althaus and Gauch, Habermacher updated the material shares used by the two authors with more recent information published in literature but also showed that, ultimately, the original material composition presented by Schweimer and Levin had not changed considerably compared to the new data (Habermacher 2011). Hence, the baseline glider dataset published in Habermacher (2011) was chosen as the reference source for the new glider production dataset. The new dataset is defined in "kg glider" so that users can easily adapt it to their weight needs. While it details a large variety of specific materials which enter the glider (e.g. various types of metals, plastics and glass), the dominant one in terms of mass is by far steel. The end of life of the glider is described in the dataset "treatment of used glider, passenger car, shredding". Here, the amount of recovered aluminium, copper, ferrous scrap, waste plastic mixture and shredder residue per kilogram of shredded glider is given. The fractions have been calculated using the transfer coefficients presented in Hischier (2007), considering the exact quantities of materials in the glider and assuming that part of the large amount of plastic in the glider can be recovered from the shredding residue since, as described in GHK \& Bio Intelligence Service (2006), post-shredding technologies for recovering other fractions are becoming more and more common. The quantities of the recovered materials are shown in Table 1.

The fractions are accounted for as "by-products/wastes" in the dataset through standard ecoinvent processes which model the recycling/disposal of the various materials. Overall, this means that this dataset (and the other treatment datasets described below for the ICE powertrain and BEV powertrain) covers the main exchanges required for reducing the glider into the various recyclable material or waste fractions. The efforts for the actual recycling or disposing of the materials are not directly included. Once the recovered fractions are indicated as by-products/wastes, their further contributions are addressed by the ecoinvent framework based on the needs of

\begin{tabular}{lll}
$\begin{array}{ll}\text { Table 1 Recovered } \\
\text { materials per kilogram } \\
\text { of shredded glider }\end{array}$ & Fraction & $\begin{array}{l}\text { Quantity per kg } \\
\text { shredded glider }\end{array}$ \\
\cline { 2 - 3 } & $\begin{array}{ll}\text { Aluminium scrap } \\
\text { Copper scrap }\end{array}$ & $4.2 \mathrm{~g}$ \\
& Ferrous scrap & $6.6 \mathrm{~g}$ \\
& Plastic & $155 \mathrm{~g}$ \\
& Residue & $180 \mathrm{~g}$ \\
\hline
\end{tabular}

the data user and particularly through his choice of the allocation model. In the modelling of the end of life of the glider, due to lack of information, the shredding process was modelled through data taken from an ecoinvent version 2 dataset describing the shredding of $1 \mathrm{~kg}$ of electronic equipment. The use of data describing the shredding of electronic equipment (meaning the material transfer coefficients as well as the infrastructure, electricity requirements, infrastructure and emissions) was assumed to be an acceptable approximation since, technically, the two processes are similar. While this is certainly an approximation, following Jody et al. (2010), vehicles are also shredded together with other appliances or scrap which seems to suggest that these facilities are actually used for a variety of products and that, therefore, the properties of shredders should be comparable. Overall, the dataset "glider production, passenger car" includes the materials (metals, plastics, chemicals, etc.), the energy (both heat and electricity), the fabrication plant, the production scrap and the emissions involved in the production of a passenger car glider and also takes into account its end of life.

\subsubsection{BEV powertrain production}

As shown in Fig. 5, the dataset powertrain production, for electric passenger car comprises the production of, respectively, the charger, the electric motor, the PDU, the converter and the inverter. The data describing the production of these devices has been obtained directly from the Swiss company Brusa which is a leading manufacturer of components for electric vehicles. The details on the data and data collection can be found in Habermacher (2011). Each of the datasets describes the specific materials (e.g. types of metals and plastics), the various electronic components (resistors, capacitors, PWBs, etc.), the production scrap and the production efforts involved in the manufacturing of $1 \mathrm{~kg}$ of each device. Based, again, on direct information from Brusa, the powertrain production, for electric passenger car dataset has been developed considering a charger of $6.2 \mathrm{~kg}$, a converter of $4.5 \mathrm{~kg}$, an electric motor of $53 \mathrm{~kg}$, an inverter of $9.5 \mathrm{~kg}$, a PDU of $3.9 \mathrm{~kg}$ and $3 \mathrm{~m}$ of copper cables through the ecoinvent process "cable, three-conductor cable". Identifying a dominant material, as was done for the glider, is not possible here since many of the materials are accounted for in the various ecoinvent datasets for the electronic components. The BEV powertrain production dataset also takes into account the end of life of the various devices through the single process "used powertrain from electric passenger car, manual dismantling". This process models the manual dismantling of the powertrain in which the bulk metal in the devices (e.g. cases or bulk metal in the electric motor) is separated from the electronics. Overall, the main fractions in which the powertrain was dismantled are shown in Table 2. The fractions have been calculated from the exact quantities of materials in the powertrain 
Table 2 Recovered fractions per kilogram of dismantled BEV powertrain

Fraction

Quantity per kg dismantled $\mathrm{BEV}$ powertrain

\begin{tabular}{ll}
\hline Aluminium scrap & $270 \mathrm{~g}$ \\
Copper scrap & $125 \mathrm{~g}$ \\
Ferrous scrap & $411 \mathrm{~g}$ \\
Electronic component scrap & $194 \mathrm{~g}$ \\
\hline
\end{tabular}

and assuming that the manual dismantling process would not lead to losses of the materials.

The outputs of this process are accounted for through standard ecoinvent by-product/waste processes describing the recycling/re-melting of the various materials. The BEV powertrain production dataset is defined in "kg powertrain" so that users can easily choose the required quantity based on the weight of the systems they need to model. As for the case of the glider, having the end-of-life dataset as an exchange in the powertrain production dataset takes from the users the burden of recalculating the amount of by-products and wastes for changing quantities of powertrain input.

\subsubsection{Internal combustion engine powertrain}

The dataset "ICE production, passenger car" has been built with a similar methodology as the one described for the glider. This means that it relies on the data presented in Schweimer and Levin (2000) and Althaus and Gauch (2010) and that it describes the materials (metals, plastics, chemicals, etc.), the energy (both, heat and electricity), the fabrication plant, the production scrap and the emissions involved in the production of a passenger car ICE powertrain (including the gears, tank, cooling and exhaust system) and also takes into account its end of life. An update of the material shares, as was done by Habermacher for the glider, was not done in this case. For the ICE powertrain, the dominant weight contributors are aluminium followed closely by steel. The end of life of the ICE powertrain has been modelled too, through a shredding process for which the same limitations expressed for the glider hold. The resulting dataset includes the shredding infrastructure and the emissions and returns the aluminium, copper, ferrous scrap, waste plastic and residue shredder fractions produced, based on the exact quantities of materials in the production dataset (Table 3 ).

These output fractions are then accounted for through standard ecoinvent by-product/waste processes. The same happens with the spent catalyst from the exhaust system of the ICE through the exchange "spent automobile catalyst" which covers the recycling of the platinum group metals. The dataset ICE production, passenger car is defined in
Table 3 Recovered fractions from the shredding of $1-\mathrm{kg}$ internal combustion engine powertrain

\begin{tabular}{ll}
\hline Fraction & $\begin{array}{l}\text { Quantity per kg dismantled internal } \\
\text { combustion engine }\end{array}$ \\
\hline Aluminium scrap & $409 \mathrm{~g}$ \\
Copper scrap & $5.7 \mathrm{~g}$ \\
Ferrous scrap & $299 \mathrm{~g}$ \\
Plastic & $135 \mathrm{~g}$ \\
Residue & $153 \mathrm{~g}$ \\
\hline
\end{tabular}

kilogram so that users can easily choose the desired amount based on their needs.

The glider and powertrain production (and end of life) datasets can then be combined to define vehicles, be it a BEV using the glider and the corresponding electric components, or some form of ICE vehicle, be it petrol, diesel or natural gas, using the glider with the ICE. Taking these datasets with the desired quantity allows users to define the vehicles which more closely correspond to their needs. As shown in Fig. 5, this is done in the new dataset structure with the passenger car production datasets.

\subsubsection{Passenger car production: electric, diesel and petrol/natural gas}

The three datasets "passenger car production, electric, without battery", "passenger car production, diesel" and "passenger car production, petrol/natural gas" essentially bring together the glider dataset and the BEV powertrain or ICE to define the specific vehicle one is interested in. For the ICE vehicles, two separate ones were defined for the diesel and petrol/natural gas vehicles as the weight of the ICE powertrain can vary for the two technologies. The same dataset is used for both petrol and natural gas vehicles since the technology of the two powertrains is basically the same, except for the pressure tank needed for natural gas vehicles which, however, is neglected in our models. The datasets also include the manual dismantling step of the vehicles. The resulting waste fractions are directly accounted for in the passenger car production datasets, due to the simplicity of this dataset and the simplicity of the manual dismantling step. The output fractions are waste glass, oil and rubber - all defined through ecoinvent by-product/waste exchanges. The manual dismantling infrastructure is modelled through a "manual treatment facility, waste electric and electronic equipment" proxy from the ecoinvent database due to lack of more detailed models. The three passenger car production datasets are defined in kilogram so that users can assess the impact of the required vehicle by simply choosing the desired amount of mass for the dataset. For how the dataset is constructed, if the mass of the vehicle is changed, the ratio between glider and drivetrain weight is kept constant, 
assuming that, in case of higher or lower weights, glider and drivetrain would scale analogously. This is of course an approximation since a slight change in mass between two vehicles from the same class could derive from various design choices. The two vehicles could have, for example, gliders with a similar weight, but one vehicle could have a more powerful motor. In the development of the datasets, it was decided to scale the glider and powertrain together to take into account that, physically, a larger vehicle will have a heavier glider which should be equipped with a more powerful powertrain in order to maintain a similar motion performance. In this context, it must also be noted though that the glider and powertrains have been modelled from data describing a compact size car. Care should therefore be taken if different car classes are modelled starting from these datasets.

The passenger car production datasets can then be used as inputs to the transport datasets which bring together all the exchanges required for describing the transport service. In this paper, only the transport dataset for the BEV will be described. The ones covering ICE passenger car transports are described in Simons (2013).

\subsection{Structure of the new electric passenger car transport dataset}

Figure 6 shows the final structure of the dataset "transport, passenger car, electric" in ecoinvent version 3 which describes the driving of $1 \mathrm{~km}$ in a compact class BEV using a lithiumion battery.

The figure shows the exchanges which appear in the transport dataset. What is also shown in Fig. 6 is how, in the new transport dataset, the amounts of some of the exchanges are defined through parameters and mathematical formulas. These are defined such that, ultimately, the amount needed to account for 1-km driving is selected. This parameterisation was introduced in order to link the choice of the amount to relevant vehicle transport characteristics. Of course, this link existed conceptually also for the entries of the transport dataset for ecoinvent version 2, but introducing the parameters highlights the link and thereby enhances the transparency of the dataset and gives the chance to users to adapt it more easily to the specific needs of their analysis. In Sect. 3, the default values given to these parameters are presented. Here, the choice of these parameters is discussed. Also, the discussion focuses on the exchanges for the passenger car, the battery, the electricity and the maintenance, as these required particular modelling considerations, whereas the road, brake ware, road ware and tyre wear entries depend on ecoinvent models defined by other data submitters. Information on the latter three can be found in Simons (2013).

The main parameters which have been defined for the transport dataset are the following: $v$ mass_without_battery, which stores the vehicle mass excluding the battery weight; vehicle life, which stores the total distance in kilometer which the vehicle will be used for during the entire use phase; battery_mass, which stores the weight of the battery; battery_life, which stores the total distance in kilometer which the battery will be used for; vehicle_energy_consumption, which describes the electric energy consumed by the vehicle for travelling $1 \mathrm{~km}$.

In the transport dataset, the exchange "passenger car, electric, without battery" takes into account the vehicle production and end of life. Since, as seen in the previous sections, the passenger car production dataset is defined in kilogram, the ratio of the desired mass of the vehicle and of the vehicle life is the amount of vehicle which is allocated to driving the BEV over $1 \mathrm{~km}$. The exchange "battery, Li-ion, rechargeable, prismatic" describes the amount of battery which is used by the vehicle. As previously described, as opposed to the version available in ecoinvent 2.2 , the battery has been taken out of the vehicle production, since it is one of the most critical components in a BEV, and having it directly in the transport dataset makes it easier for users to change its quantity or even type. The formula which defines the amount can be thought as of composed of two parts. The first one, battery_mass/vehicle_life, describes the contribution for $1 \mathrm{~km}$ of the battery in the vehicle, as for the vehicle. The second one, vehicle_lifel battery_life, is a multiplication factor which takes into account

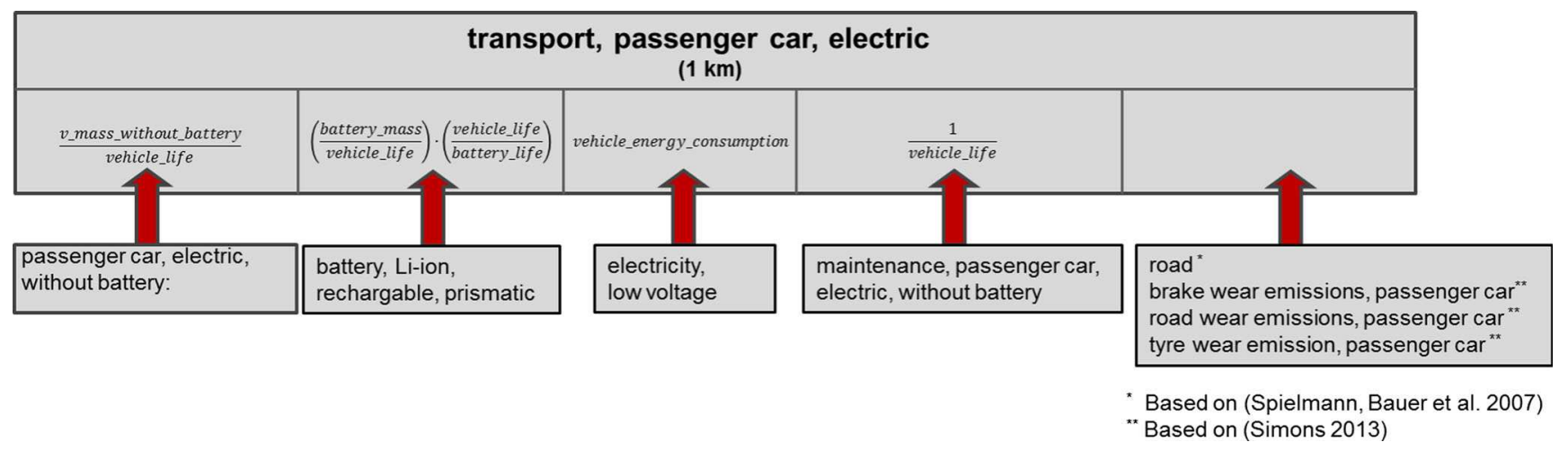

Fig. 6 Scheme of the electric passenger car transport dataset defined for 1-km driving 
possible battery exchanges due to maintenance. If the battery life is shorter than the vehicle life, the value will be larger than 1. Further, since this dataset describes an average of transports with electric vehicles, this value can be a non-integer number since for some vehicles, a change due to maintenance might be necessary, while for others not.

The "electricity, low-voltage" exchange describes the electric energy which on average needs to be provided from the low-voltage grid to charge the vehicles battery for travelling over $1 \mathrm{~km}$. Charging and discharging losses and all losses in the powertrain are thus taken into account. A "low-voltage" electricity input has been chosen assuming that most of the vehicles today will be charged at low-voltage stations since fast charging, high-voltage stations are not, as can be seen, for example, in Trigg and Telleen (2013), yet widely available. The user can define the consumption through the parameter vehicle_energy_consumption. Overall, the energy consumption of an electric vehicle depends on a large number of variables including the mass of the vehicle, the efficiency of the powertrain and battery, the road type over which it travels (e.g. urban vs mountain road), the driving behaviour, traffic conditions or the use of auxiliaries like lights or heating and cooling devices due to meteorological conditions, and the values typically reported in literature have strong variations (Hawkins et al. 2012a). To overcome these variations, sometimes, the consumption of vehicles is calculated within the framework defined through specific standards. One of the most utilised standards is the New European Driving Cycle (NEDC), even though, as described in Mock et al. (2012), there is evidence that the NEDC underestimates the consumption of vehicles in real life operation. Since the electric passenger transport dataset should describe an average transportation with a BEV, the focus was set on estimating an average real-world consumption. This was done using the methodology proposed in Althaus and Gauch (2010), in which the consumption of the vehicle is obtained through following steps. First, the mechanical energy required to move the mass of the vehicle along the trajectory defined by the NEDC is calculated. The mechanical energy required to move a vehicle can be calculated by integrating along the path of motion the equation describing the dynamics of a road vehicle, shown, for example, in (Guzzella and Sciarretta 2005):

$$
F(t)_{t}=m_{v} a(t)+\frac{1}{2} \cdot c_{w} \cdot \rho \cdot A \cdot v^{2}(t)+c_{r} \cdot m_{v} \cdot g
$$

where $F(t)_{t}$ is the force required for the traction of the vehicle, $m_{v}$ is the mass of the vehicle, $a(t)$ and $v(t)$, respectively, are its acceleration and speed, $c_{w}$ is the aerodynamic drag coefficient, $A$ the frontal area, $\rho$ the density of the air, $c_{r}$ the rolling resistance and $g$ the acceleration due to gravity. After integrating the equation above over the speeds and accelerations defined by the NEDC in order to obtain the required mechanical energy for the motion, the corresponding electrical energy is obtained by multiplying the mechanical energy with the average efficiency of the entire powertrain chain, meaning the efficiency of the power electronics, the internal charging and discharging efficiency of the battery and the efficiency of the charger. Following Habermacher (2011), the average efficiency of the entire electric powertrain was set to around $70 \%$ including the losses deriving from the charger, while for those parts of the NEDC which model an urban driving condition, an increase of $5 \%$ in the efficiency was considered due to the energy recovered through regenerative braking. Also, still based on the methodology proposed by Althaus and Gauch (2010), an increment on the resulting energy of $15 \%$ was added in order to take into account the higher consumption typically experienced in real-life driving as opposed to the driving style and trajectory imposed by the NEDC. In addition to that, consumption contributions deriving from cabin heating, air-conditioning (cooling) and the use of other equipment like lights, radio or windscreen wipers have also been added. For heating, direct electrical resistance heating has been assumed, but it should be noted that it is expected, as described for example in Duleep et al. (2011), which future heating systems for BEVs will be based on heat pumps due to their lower consumption. Following Althaus and Gauch (2010), the added consumption due to auxiliaries was computed assuming an average speed of $50 \mathrm{~km} / \mathrm{h}$ and a nominal power for heating, air-conditioning and lights, radio, etc., of, respectively, $3,0.6$ and $0.5 \mathrm{~kW}$. This ultimately leads to a contribution for heating and cooling of, respectively, 2 and $0.4 \mathrm{kWh} / 100 \mathrm{~km}$ (assuming that each of them is switched on over a period of 4 months during the year) and for lights, radio, etc., of $0.33 \mathrm{kWh} /$ $100 \mathrm{~km}$ (with this equipment being switched on on $33 \%$ of the journeys). The default energy consumption value which has been obtained for the new ecoinvent electric passenger car transport dataset will be presented in Sect. 3. Figure 7 summarises the main steps of the consumption calculation model.

The maintenance of the vehicle has been modelled taking the maintenance dataset for a conventional vehicle from $v 2.2$ of the ecoinvent database and removing the exchanges corresponding to the maintenance of the lead-acid battery and to the oil replacement, assuming that for both vehicle technologies, the remaining parts (e.g. tyres, plastic and steel pieces) would suffer the same deterioration. The dataset describes all the exchanges required over the entire use phase of the vehicle. Again, since the transport dataset is defined for $1 \mathrm{~km}$, the amount of maintenance accounted for equals the inverse of the vehicle life. As previously explained, the maintenance contribution due to a battery exchange is directly taken into account in the exchange for the amount of battery in the transport dataset and is not included in the maintenance dataset.

With respect to the exchanges due to road and non-exhaust emissions, these are accounted for through standard ecoinvent 


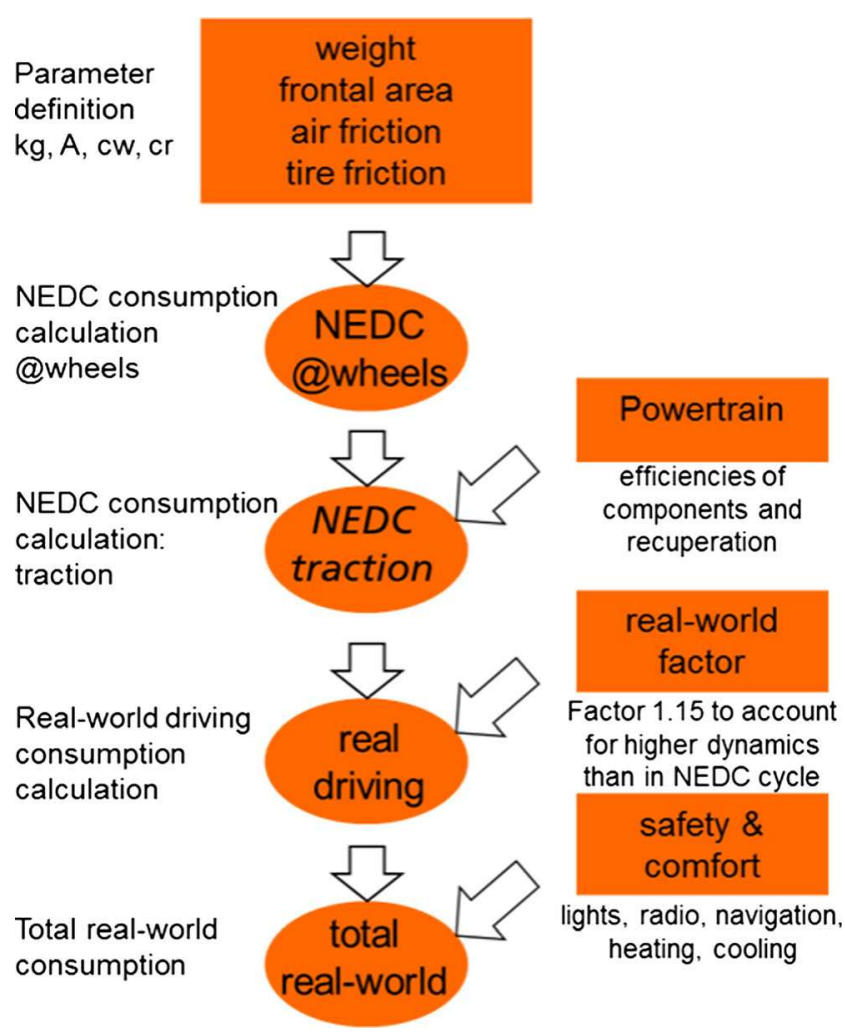

Fig. 7 Consumption calculation model

datasets. Non-exhaust emissions are split according to tyre wear, brake wear and road abrasion and are modelled through the new models by A. Simons (Simons 2013). As described in Simons' paper, their amounts are defined through specific parameters and formulas and ultimately scale with the weight of the vehicle. The only difference in the electric passenger car transport dataset as opposed to the models given by Simons is that only $20 \%$ of the brake wear emissions caused by ICE vehicles are considered for BEVs since, as described in Althaus and Gauch (2010), due to regenerative braking, mechanical brakes are used considerably less. This is accounted for in the mathematical formula defined for the brake wear emissions with the multiplying factor of 0.2. In Althaus and Gauch (2010), only $10 \%$ of the brake wear emissions were considered. Since at the time of writing, to the best of the authors' knowledge, no further data on brake wear reduction in electric passenger cars could be found, it was decided to make the more conservative choice of $20 \%$.

\section{Results}

3.1 Default values of the transport dataset

The final electric passenger car transport dataset published in ecoinvent version 3 has the geographical location GLO (global). For now, no country-specific electric passenger transport dataset was submitted. Table 4 shows the default values of the parameters used to define the amount of exchanges in the dataset.

For the vehicle life, $150,000 \mathrm{~km}$ is used, a conservative value in accordance with the ones typically reported in literature, as shown, for example, in (Hawkins et al. b). Following the results presented in Habermacher (2011), the mass of the vehicle without battery was set to $918 \mathrm{~kg}$ and the one of the battery pack, without the maintenance contribution, to $262 \mathrm{~kg}$. These values are in range with the ones of other compact class vehicles described in Grünig et al. (2011). The mass of the vehicle without battery originates from $838 \mathrm{~kg}$ glider and about $80 \mathrm{~kg}$ of electric drivetrain. Following Althaus and Gauch (2010), the consumption of the vehicle was calculated based on the aerodynamic characteristics defined through the parameters shown in Table 5.

With the total mass of the vehicle of 1,250 (including $75 \mathrm{~kg}$ for a driver), the final real-world consumption for the vehicle is of $0.199 \mathrm{kWh}$ for 1-km driving (Habermacher 2011). This value leads to a range of around $120 \mathrm{~km}$, with the battery mass of $262 \mathrm{~kg}$ and a battery energy density of $114 \mathrm{Wh} / \mathrm{kg}$, which is the one of the lithium-ion battery currently available in the ecoinvent database. To date, there is a strong debate on reasonable values for the life expectation of batteries (Hawkins et al. 2012a), and historical data for current technologies is missing. As in Althaus and Gauch (2010) and Habermacher (2011), it was assumed that on average, $50 \%$ of the vehicles would need a battery replacement during the use phase which leads to a battery life parameter of 100,000.

In order to get a feeling about the numerical results produced by this dataset, its GHG emissions from the default ecoinvent allocation option (allocation at the point of substitution) with version 3.01 of the database have been compared to the recent results shown in Hawkins et al. (2012b) and Hawkins et al. (2013) in which, building on the glider model from the GREET 2.7 vehicle cycle model (Burnham et al. 2006) and configuring the BEV powertrain starting from a Nissan Leaf, the global warming potential (GWP) for the production of various BEVs is presented. In Hawkins et al. (2013), a contribution from vehicle production in the range of 72 to $81 \mathrm{~g} \mathrm{CO}_{2}$ equivalents per $\mathrm{km}$ is reported in this context.

Table 4 Default values of the parameters in the electric passenger car transport dataset

\begin{tabular}{ll}
\hline Parameter & Default value \\
\hline Vehicle life & $150,000 \mathrm{~km}$ \\
Vehicle mass without battery & $918 \mathrm{~kg}$ \\
Battery mass & $262 \mathrm{~kg}$ \\
Battery life & $100,000 \mathrm{~km}$ \\
Vehicle energy consumption & $0.199 \mathrm{kWh} / \mathrm{km}$ \\
\hline
\end{tabular}


Table 5 Aerodynamic characteristics of the modelled vehicle

\begin{tabular}{ll}
\hline Parameter & Default value \\
\hline Frontal area $A$ & $2.2 \mathrm{~m}^{2}$ \\
Aerodynamic drag coefficient $c_{w}$ & 0.31 \\
Rolling resistance $c_{r}$ & 0.01 \\
\hline
\end{tabular}

Table 6 shows the key exchanges of the new ecoinvent version 3 electric passenger car transport dataset together with the corresponding GWP contributions (GWP hundred years; (IPCC 2007))

First of all, in analyzing the results in Table 6 , it has to be noted that these are for a global dataset, which means that the electricity exchange is accounted for by the ecoinvent version 3 framework as a global, low-voltage mix, and this leads to the very high contribution. If, for example, a Swiss low-voltage mix was used (see Treyer and Bauer 2013) for details on the electricity datasets in ecoinvent version 3 ), the contribution would be around $28 \mathrm{~g} \mathrm{CO}_{2}$ equivalents per $\mathrm{km}$ and the total impacts around 110 grams $\mathrm{CO}_{2}$ equivalents per km. Below, we will focus on the GWP of the submitted datasets. As can be seen from Table 6, the GWP value for the vehicle only, which comprises the production of the vehicle without battery, the battery including maintenance and the vehicle maintenance compares very well with the results presented in Hawkins et al. (2013). Taking the normalised GWP value reported in the supporting information of Hawkins et al. (2013) for the "base vehicle" (which corresponds to what is termed glider in this paper) incremented by the value for the end of life and comparing it to the sum of the emissions from all the contributors in the vehicle production (i.e. vehicle base, end of life, engine, other powertrain, battery, use-phase non-fuel-related) suggests that the contribution of the production and end of life of the glider account for about 42 to $46 \%$ compared to the entire vehicle depending on the battery type considered. Doing the same for the electric motor and other powertrain components leads to a contribution of around 10 to $11 \%$.
Analysing the specific GWP contributions obtained with the default ecoinvent allocation option for the glider and electric powertrain discussed in this paper, one obtains, respectively, $0.039 \mathrm{~kg} \mathrm{CO}$ equivalents and $0.0098 \mathrm{~kg} \mathrm{CO}$ equivalents, which corresponds to around 52 and $13 \%$ of the GWP emissions of the entire vehicle production and end of life, including the battery. These results are higher, but overall comparable to those presented by Hawkins et al.

\subsection{Market datasets}

When browsing the database, users will find, next to the datasets described above, other dataset which are automatically generated by the new ecoinvent version 3 framework. These datasets describe market activities and appear in the database with names starting as "market for" followed by the name of the dataset for which the market has been defined. For example, in addition to the datasets "transport, passenger car, electric" or "passenger car production, electric, without battery", users will find the datasets "market for transport, passenger car, electric" and "market for passenger car electric, without battery". As described in more detail in Weidema et al. (2013), the function of market datasets is to provide users with datasets describing consumption mixes and the other activities (like transport or wholesaler and/or retailer activities) required to define the availability of a class of products on a market. At the time of writing, only one dataset has been submitted for each of the products or activities related to the electric passenger transport dataset or the datasets related to the production of the passenger car, and all these are defined as GLO geography. To date, users will therefore find, for each of the datasets, only one global market dataset which ultimately links to the corresponding single transport or production dataset. If, for example, new geography locations are added for these datasets in the future, these will be linked to the corresponding market activities. The reader is referred to the ecoinvent version 3 data quality
Table 6 Main exchanges of the global electric passenger car transport dataset and greenhouse gas emissions

\begin{tabular}{llll}
\hline Transport, passenger car, electric & $1 \mathrm{~km}$ & & \\
Exchanges & Quantity & Unit & $\mathrm{kg} \mathrm{CO}_{2}$ equivalents/km \\
Battery, Li-ion, rechargeable, prismatic & $2.62 \mathrm{E}-03$ & $\mathrm{~kg}$ & $1.63 \mathrm{E}-02$ \\
Electricity, low voltage (global) & $1.99 \mathrm{E}-1$ & $\mathrm{kWh}$ & $1.58 \mathrm{E}-01$ \\
Maintenance, passenger car, electric, without battery & $6.67 \mathrm{E}-06$ & $\mathrm{unit}$ & $8.10 \mathrm{E}-03$ \\
Passenger car, electric, without battery & $6.12 \mathrm{E}-03$ & $\mathrm{~kg}$ & $5.02 \mathrm{E}-02$ \\
Road & $4.87 \mathrm{E}-04$ & $\mathrm{~m} *$ year & $5.74 \mathrm{E}-03$ \\
Brake wear emissions, passenger car & $1.05 \mathrm{E}-06$ & $\mathrm{~kg}$ & 0 \\
Road wear emissions, passenger car & $1.16 \mathrm{E}-05$ & $\mathrm{~kg}$ & 0 \\
tyre wear emissions, passenger car & $6.76 \mathrm{E}-05$ & $\mathrm{~kg}$ & 0 \\
Total & & & $2.39 \mathrm{E}-1$ \\
Vehicle only & & & $7.46 \mathrm{E}-2$ \\
\hline
\end{tabular}


guidelines (Weidema et al. 2013) for more information on the use of market activities.

\subsection{Limitations}

The biggest limitation in the submitted datasets lies in the main data source describing the production of the glider and, particularly, of the ICE powertrain. While the analysis presented in Schweimer and Levin (2000), which laid the foundations for the glider and ICE powertrain datasets discussed above, is extremely detailed and represents an invaluable wealth of information, it nevertheless describes the production of a vehicle in the year 2000. As described, for example, in Castro et al. (2003), there is a trend towards a higher use of lightweight materials in the automotive industry in order to reduce the overall weight of vehicles. This means that, ultimately, in the new datasets, the share of lightweight materials like polymers or non-ferrous alloys might be underestimated in comparison to current vehicle technology. Since the dataset for the production of the glider originates from the results presented in Habermacher (2011) which include an update of the material shares from Schweimer and Levin (2000) based on more recent literature, this problem affects the ICE powertrain dataset more than the one of the glider. At the same time, in Habermacher (2011), it is shown that there are differences between the updated shares of materials and the ones reported in Schweimer and Levin (2000) for the Golf A4, but that these differences are relatively small, highlighting that there is a trend towards different materials, but that this has not yet manifested itself with drastic changes. For this reason, it is believed that the new glider and ICE powertrain datasets are still suitable for current technologies. On the other hand, a thorough, complete and transparent analysis like the one made by Schweimer and Levin in 2000 is not available at the moment for state-of-the-art passenger car production processes to the best of the authors' knowledge. In the light of the discussed trends in material changes, such a study would represent an essential base for the analysis of nextgeneration transport systems. This highlights the need for more research in the area of life cycle inventory data of vehicle production. Moreover, if electric vehicles are to play a key role in the transition towards more sustainable transport systems, then renewable and clean electricity sources need to be used for battery charging. As shown, for example, in Althaus and Gauch (2010) or Hawkins et al. (2012a), under this scenario, the largest impacts typically derive from the production of the vehicle (including the battery) for most impact indicators. This is therefore the sector in which the data with the highest quality will be needed and in which the largest potentials for improving the sustainability of electric passenger transport will lie. Hence, an ongoing update of the vehicle production datasets addressing, for example, the use of lightweight materials will be required.
With respect to the limitations in the practical use of the datasets, two main observations can be made. First, it needs to be remembered that the datasets have been derived from data describing a compact class vehicle. The ratio of glider and drivetrain is therefore optimised for that class. Using the dataset for other vehicle classes simply considerably increasing or decreasing the total vehicle mass may lead to imprecise results. Second, care must be taken when changing the parameters used to define the amount of the exchanges in the transport dataset (Table 4). There are no mathematical relations linking the various parameters in the different exchanges in the dataset but changing one parameter could lead, in a realistic transport situation, to changes in other ones. When changing the mass of the vehicle, for example, the user should consider that a heavier vehicle will also require more energy for the motion and a larger amount of battery to achieve a given range. Hence, the masses, consumption and life times can be changed, but it is up to the user to use a consistent methodology which correctly models the specific situation of interest. Finally, it should also be noted that the degree to which the introduced parameterisation will be of support to users will ultimately also depend on how software tools will integrate the new database.

\section{Conclusions}

In this paper, the new electric passenger car transport dataset and the ensemble of related datasets which are included in version 3 of the ecoinvent database have been presented. The transport dataset describes the transport over $1 \mathrm{~km}$ with a compact class electric passenger car. Next to the transport dataset, datasets describing the production, end of life and maintenance for an electric passenger car have been developed. Specific vehicle production and end-of-life datasets have also been generated for ICE diesel and petrol/natural gas vehicles and are discussed. The datasets have been developed with a strong modular approach, defining a hierarchy of datasets which address various levels of functionality in the vehicle. This allows to describe the production and end of life of the vehicle by linking together various datasets addressing the materials and efforts involved in the production and end of life of specific vehicle components, strongly enhancing the flexibility of the vehicle database structure as opposed to the previous datasets for ecoinvent version 2.2. Thanks to this modularity it is possible, on one side, to quantify more easily the impacts deriving from the various components in a vehicle and, on the other, to build virtual vehicles through the now available library of datasets on vehicle components. While the data for the ICE passenger car production mainly originates from the same sources used in the previous version of the ecoinvent database, the data describing the production of the 
electric motor and of the power electronics in the electric vehicle is new and was obtained from a leading manufacturer of state-of-the-art components for BEVs. Next to modularisation, parameters and mathematical formulas were introduced in the electric passenger car transport dataset for defining part of its exchange quantities. The parameters and formulas relate the choice of specific exchange amounts to key transport characteristics, giving users the chance to adapt more easily the default transport dataset to their needs in terms of vehicle mass, vehicle and battery life times and vehicle consumption. Overall, this enhances the transparency of the transport dataset compared to its previous versions. The relative contributions in GWP obtained from the datasets describing the two main vehicle components in the BEV have been compared to results presented by other authors in a recently published study, and good agreement was found.

The paper ends with a discussion on the limitations of the new datasets, identifying the glider production dataset and the ICE production dataset as the more critical ones due to the older age of the data these are based on. Overall, if BEVs are to play a dominant role in the transition towards more sustainable transport systems, then renewable and clean electricity sources need to be used to limit the impacts from the use phase. Since, in this scenario, the main impacts lie in the production of the vehicle, this highlights the need for further research in the area of LCI data of vehicle production and for an ongoing update of vehicle production and end-of-life datasets.

Acknowledgments We are thankful to our colleagues at the Paul Scherrer Institute Christian Bauer and Andrew Simons for valuable support.

\section{References}

Althaus HJ, Gauch M (2010) Vergleichende Ökobilanz individueller Mobilität: Elektromobilität versus konventionelle Mobilität mit Bio- und fossilen Treibstoffen. Empa, Swiss Federal Laboratories for Materials Science and Technology, Report available from http:// www.empa.ch/plugin/template/empa/*/104369/—/l=1. Retrieved from http://www.empa.ch/plugin/template/empa/*/104369/_/l=1

Burnham A, Wang M, Wu Y (2006) Development and applications of GREET 2. 7 - The Transportation Vehicle-Cycle Model

Castro MG, Remmerswaal JM, Reuter M (2003) Life cycle impact assessment of the average passenger vehicle in the Netherlands. Int J Life Cycle Assess 8(5):297-304

Duleep G, van Essen H, Kapman B, Grünig M (2011) Assessment of electric vehicle and battery technology. Report by CE Delft and Ecologic; commissioned by the European Commission within the study: Impacts of electric vehicles - deliverable 2. Retrieved from www.cedelft.eu/

Frischknecht R, Jungbluth N, Althaus HJ, Doka G, Dones R, Heck T, Wernet G (2007). Overview and methodology-data v2.0. Ecoinvent Report no.1

GHK, \& Bio Intelligence Service (2006) A study to examine the benefits of the End of Life Vehicles Directive-Final Report to DG Environment
Grünig M, Witte M, Marcellino D, Selig J, van Essen H (2011) An overview of electric vehicles on the market and in development. Report by CE Delft and Ecologic; commissioned by the European Commission within the study: Impacts of electric vehicles - deliverable 1. Retrieved from www.cedelft.eu/

Guzzella L, Sciarretta A (2005) Vehicle propulsion systems. Springer (ed)

Habermacher F (2011) Modeling material inventories and environmental impacts of electric passenger cars. Master Thesis, Department of Einvironmental Sciences, ETH Zurich

Hawkins TR, Gausen OM, Stromman AH (2012a) Environmental impacts of hybrid and electric vehicles - a review. Int J Life Cycle Assess 17(8):997-1014

Hawkins TR, Singh B, Majeau-Bettez G, Stromman AH (2012b) Comparative environmental life cycle assessment of conventional and electric vehicles. J Ind Ecol 17:53-64

Hawkins TR, Singh B, Majeau-Bettez G, Stromman AH (2013) Corrigendum to: Hawkins, T. R., B. Singh, G. Majeau-Bettez, and A. H. Strømman. 2012. Comparative environmental life cycle assessment of conventional and electric vehicles. Journal of Industrial Ecology doi:10.1111/j.1530-9290.2012.00532.x. J Ind Ecol 17(1):158-160

Hischier R (2007) Disposal of electric and electronic equipment (ewaste), ecoinvent report nr. 18, Part V

Huggins, D (2009) Panorama of Transport, Eurostat Statistical Books, Published by the European Commission.

IPCC (2007) IPCC Fourth Assessment Report: Climate Change 2007. Intergovernmental Panel on Climate Change

Jody EJ, Duranceau CM, Pomykala JA, Spangenberger JS (2010) Endof-life vehicle recycling: state of the art of resource recovery from shredder residue

Mahieu, Y. (2009). "Highlights of the Panorama of Transport." Eurostat Statistics in Focus 42.

Mock P, German J, Bandivadekar A, Riemersma I (2012) Discrepancies between type approval and "real-world" fuel consumptionand CO2 values. ICCT Working Paper, 2012-02

Schneider J, Karigl B, Neubauer C, Tesar M, Oliva J, Read B (2010) End of life vehicles: legal aspects, national practices and recommendations for future succesful approach

Schweimer GW, Levin M (2000) Life Cycle Inventory of the Golf A4. Research Environment and Transport Volkswagen AG, Wolfsburg and Center of Environmental System Research, University of Kassel, Germany

Simons A (2013) Road transport: new life cycle inventories for fossil fuelled passenger cars and non-exhaust emissions in ecoinvent v3. Int J Life Cycle Assess. doi:10.1007/s11367013-0642-9

Spielmann M, Bauer C, Dones R, Tuchschmid M (2007) Transport services. Ecoinvent Report No. 14

THELMA (2010) Technology-centered Electric Mobility Assessment http://www.thelma-emobility.net/

Treyer K, Bauer C (2013) Life cycle inventories of electricity generation and power supply in version 3 of the ecoinvent database - part II: electricity markets. Int J Life Cycle Assess. doi:10.1007/s11367013-0694-x

Trigg T, Telleen P (2013) Global EV outlook-report supported by Clean Energy Ministerial, Electric Vehicles Initiative and International Energy Agency

Volkswagen AG (2008) The Golf-environmental commendation-detailed version. Retrieved from http://www.volkswagenag.com/content/ vwcorp/info center/en/publications/2008/04/environmental commendation0.-bin.acq/qual-BinaryStorageItem.Single.File/GolfUP_Hintergrundbericht_GB.pdf

Weidema BP, Bauer C, Hischier R et al (2013) Overview and methodology-data quality guidelines for the ecoinvent database version 3 